\title{
EL REGRESO DEL EXPERIMENTADOR Y LA METODOLOGÍA DE LA APERTURA DE CAJAS NEGRAS
}

\author{
Julio C. ARMERO \\ Facultad de Filosofia. UNED
}

ABSTRACT: The experimenter's regress is one of the arguments that seem to back epistemological relativism. It has been noted that it carries a close resemblance to the hermeneutical circle. This paper tries to show how the hindrances for scientific practice involved in the situation described by the argument appear in unrelated areas of research and how the difficulties are dealt with. The comparative approach seems to throw some light on a few features of baconian science often made dependent upon wide sociological fact.

El relativismo epistemológico, que se mantiene casi como ingrediente definitorio del área en numerosas clases de estudios sobre la ciencia, se caracteriza por rechazar la pertinencia y la utilidad de los objetivos y métodos de la filosofía de la ciencia tradicional, que prestaba particular atención a la lógica de los diversos aspectos de las construcciones científicas y, en especial, a la lógica de las argumentaciones involucradas en la elección de teorías.

Este relativismo se ha fundado en varios argumentos filosóficos que han captado la atención de la comunidad de los filósofos de la ciencia a lo largo del siglo XX.

Según este orden, fue el primero la tesis de Duhem y Quine, de acuerdo con la cual una falsación de una teoría nunca podría ser conclusiva, porque las teorías son sometidas a falsación bajo diversas hipótesis acerca de las condiciones en que la contrastación tiene lugar. Además, otras ideas teóricas no implicadas a primera vista en la contrastación, pueden ser retocadas si es muy ferviente el interés en mantener la teoría objeto de una contrastación negativa. 
Si las teorías que sostenemos no están sometidas exclusivamente a la contrastación empírica, sino que otro tipo de consideraciones entran en aceptarlas o rechazarlas, entonces hay una infradeterminación empírica de las teorías. Mientras que Duhem y Quine aludían entre esas otras consideraciones a la parsimonia o a la economía de los principios, los partidarios de enfoques sociológicos no dudaron en sugerir intereses más mundanos o formas de decisión basadas en la negociación y otras dinámicas sociales.

En segundo lugar, se prestó atención a la idea de que los enunciados empíricos que han de servir de base a la contrastación no son autónomos con respecto a las teorías, sino que el valor de verdad que se les reconozca e incluso el lenguaje en que vienen expresados, depende de las teorías.

Los positivistas lógicos ya habían advertido que los enunciados de la base empírica no siempre son obtenidos mediante la observación directa, sino que muy frecuentemente se emplean instrumentos de observación o de medida.

Los positivistas lógicos hablaban de esta clase de instrumentos como de extensiones de los sentidos humanos, aunque a veces reconocían que su uso y la habilidad para reconocer los resultados obtenidos con ellos entrañan lo que después se vino a llamar "carga teórica». ${ }^{1}$ Respecto a que determinados fenómenos solo tuvieran lugar si eran producidos con la ayuda de instrumentos, los positivistas lógicos no encontraban nada filosóficamente llamativo. Una generalización empírica, según ellos, se expresa mediante un enunciado universal condicional, sea o no el antecedente instanciado por una intervención humana o por el curso de la naturaleza.

De todas formas, estudiaron, siguiendo a Bridgman, aunque dentro del contexto más general de la lógica de los términos disposicionales, la lógica de los términos cuya legitimidad está garantizada por una operación controlada.

A pesar de que la doctrina de Bridgman era conocida como "operacionalismon y de que se aludía con frecuencia a la doctrina instrumentalista, que compara o identifica a las teorías con instrumentos, cuando, en filosofia de la ciencia,

${ }^{1}$ SPECTOR (1966) lleva a cabo una buena discusión del concepto de observabilidad en el positivismo lógico. 
los representantes del giro historicista entraron en polémicas con positivistas y falsacionistas, los instrumentos científicos quedaron fuera de la discusión. Merece la pena explicar por qué.

Los positivistas lógicos tenían en su origen un proyecto de fundamentar el conocimiento sobre las bases de la experiencia y la lógica. La teoría de modelos no había aparecido en su época e incluso la creación de la semántica tuvo lugar en pleno apogeo del positivismo lógico. La necesidad de nociones precisas para la metateoría fue advertida por Tarski a finales de los años veinte. De modo que adoptaron con naturalidad la idea de que las teorías eran esencialmente conjuntos de enunciados cuya propiedades lógicas debían ser estudiadas en el contexto de la teoría de los sistemas axiomáticos. En lo concerniente a la base empírica, los términos básicos deberían referirse a experiencias singulares. Ese proyecto sufrió varios tropezones y cambios, sobre todo en las propiedades exigidas a la base empírica y al análisis de las relaciones entre sus términos y el resto de la teoría. Al final se demostró que era impracticable el proyecto. Pero perduró la idea de que debería haber una clara separación entre el lenguaje teórico y el lenguaje empírico en que se habían de formular los enunciados fundamentadores.

Autores como Kuhn y Hanson trataron esta distinción y la cuestionaron del modo más radical. Que los instrumentos científicos podían incorporar carga teórica se dio por hecho y pasaron a intentar demostrar que la mera y despojada observación también está configurada por las teorías o paradigmas o visiones del mundo de los observadores. Al llevar la discusión al terreno básico de la observación, discutir los instrumentos resultaba inútil.

Bogen y Woodward ${ }^{2}$ (1992) han criticado en un extenso artículo el terreno en que tuvo lugar la polémica entre Churchland y Fodor acerca de la carga teórica de las observaciones. Según Bogen y Woodward, el conocimiento perceptivo rara vez desempeña un papel en la contrastación de las teorías. Pero lo cierto es que en esos términos plantearon la discusión de la base empírica

2 P. M. ChurChland, "Perceptual Plasticity and Theoretical Neutrality: A Reply to Jerry Fodor", Philosophy of Science, 1988, 55, pp. 167-187; J. FODOR, “A Reply to Churchland's 'Perceptual Plasticity and Theoretical Neutrality'", Philosophy of Science, 55, 1988, pp. 188-198. La cuestión de si existe una autonomía o encapsulamiento de los datos sensoriales se ha planteado con mucha anterioridad. Al menos en Husserl según la opinión de FOLLESDAL, "Phenomenology", en E. C. CarteretTe y M. P. Friedman, Haindbook of Perception, Nueva York, Academic Press, 1974. 
Hanson y Kuhn, pasándose de la diana, porque ya no quedaba en la filosofia de la ciencia nadie que defendiera el fenomenalismo. Según ellos el recurso a la contrastación empírica no puede zanjar un desacuerdo entre tradiciones teóricas rivales, entre otras cosas porque incluso la percepción está sesgada por la teoría que ha adquirido el observador a través de un proceso de adoctrinamiento. Kuhn procedió además, haciendo uso de las ideas de Quine sobre la traducción, a un análisis de los inconvenientes lingüísticos de la comparación entre tradiciones teóricas.

La discusión de los instrumentos con vistas a la defensa de intereses filosóficos fue posterior, y el argumento filosófico que les concedió una relevancia específica en la defensa del relativismo epistemológico lo ideó H. Collins (1985). Una de las formulaciones que él hace del argumento tiene lugar en el contexto de su discusión del supuesto detector de ondas de la energía gravitatoria.

En una versión más general que la que Collins ofrece, el resultado correcto de un experimento de detección de un acontecimiento ( $u$ objeto) $A$ en un lugar $\mathrm{L}$ depende de si $\mathrm{A}$ está presente en el lugar $\mathrm{L}$ donde se intenta detectar. Por lo tanto, para saber si el resultado de un experimento de detección de $A$ en $\mathrm{L}$ es correcto, debemos saber si A está presente en $\mathrm{L}$. Pero para saber si A está presente en $L$, debemos saber que hemos hecho un experimento correcto de detección de $\mathrm{A}$ en $\mathrm{L}$.

Los instrumentos entran en juego porque en la ciencia moderna muchos experimentos de detección se llevan a cabo mediante instrumentos, de modo que para saber si el experimento de detección da un resultado correcto, debemos contar con un buen detector $\mathrm{D}$ de $\mathrm{A}$. Evidentemente, para saber si hemos construido un buen detector $\mathrm{D}$ de $\mathrm{A}$ debemos saber cuál es el resultado correcto del experimento.

Esto es un círculo vicioso, y si intentamos romper el círculo proponiendo un segundo experimento de detección que sea una réplica del primero, entonces obtenemos un regreso infinito, porque para saber si el resultado del segundo experimento es correcto, debemos saber si A está presente en $\mathrm{L}$.

Collins hace un comentario que atribuye una causa al hecho de que no podamos saber si el resultado del experimento es correcto. Esta es que la experimentación es asunto de "skillful practice», o sea, de pericia práctica. Dado que la 
experimentación es asunto de pericia, nunca puede estar claro si el segundo experimento se ha hecho lo bastante bien como para contrastar la corrección del primer experimento.

Este conjunto de reflexiones es lo que se conoce como «el regreso del experimentador", que constituye una de las bases argumentativas del giro sociológico relativista en los estudios sobre la ciencia, porque de acuerdo con estos autores en la práctica el regreso es detenido mediante la negociación de la comunidad científica, que decide que un experimento ha tenido éxito o un detector detecta.

Un círculo semejante se presenta en investigaciones no relacionadas con los instrumentos científicos ni con la pericia práctica, de modo que no me voy a ocupar de las especiales complicaciones que trae consigo el asunto de la pericia práctica.

Pierre Duhem (1906), cuyo proyecto filosófico no consistía ni mucho menos en procurar la fundamentación de la ciencia, ya había reunido el tema de la infradeterminación empírica de las teorías y el de la interpretación teórica que portan los enunciados contrastadores de las teorías.

Según él, un experimento en física consiste en la observación de un grupo de fenómenos acompañada de una interpretación de estos fenómenos. La observación de los fenómenos suministra datos concretos y la interpretación reemplaza estos datos "por las representaciones abstractas y simbólicas que les corresponden en virtud de teorías admitidas por el observador». (p. 90)

El resultado, para el experimentador, de la experiencia física es un enunciado que expresa las representaciones que según la teoría corresponden a los datos concretos.

Cuando en la experiencia física se hace uso de un instrumento, éste es sustituido por una representación abstracta de acuerdo con una teoría física, de modo que sus operaciones se puedan expresar en la teoría, y los datos concretos que suministra puedan ser sustituidos por su interpretación teórica.

Sin embargo, no siempre los instrumentos son sustituidos por una representación abstracta: la bomba de aire de Boyle o el calorímetro de Lavoisier fueron presentados con muy detallados y realistas dibujos y los dispositivos experimen- 
tales de Faraday estaban descritos con gran minuciosidad. Sin remitirse a la reciente bibliografía de estudios sobre el uso de imágenes en la ciencia, es evidente que lo que dice Duhem no es cierto para una gran cantidad de casos

Dice célebremente Turner que los instrumentos son "ideas in brass" y en una amplia definición de lo que es un instrumento científico desenvuelve esta afirmación (Turner, 1993): son aparatos que incorporan el conocimiento científico que una cultura posee en un momento dado. Por otro lado dice que los instrumentos como tales son neutrales.

Las dos afirmaciones son convincentes y parecen mutuamente contradictorias. En un sentido, la determinada disposición del metal que constituye un aparato científico es neutral con respecto a cualquier teoría, claro. Un instrumento es asunto controvertible cuando alguien dice qué hace. Una multitud de aparatos ingeniosamente configurados por sus inventores solo adquiere su sentido (o sinsentido) cuando estos afirman que son máquinas de movimiento perpetuo.

L. Roberts (1991) ha estudiado cómo un aparato que en su primera presentación pública fue denominado simplemente "máquina" llegó a llamarse "calorímetron. Dejar una máquina sin nombre es situarla en un terreno menos comprometido que si se dice cuáles son sus funciones.

La misma palabra "instrumento" no sugiere un único concepto, sino más bien una diversidad de estereotipos. Ateniéndose a lo que ordinariamente se dice de los instrumentos científicos y los tipos de funciones que pueden realizar, las sustancias químicas podrían ser consideradas instrumentos: disolventes, indicadores y reactivos desempeñan funciones que normalmente se atribuyen a los instrumentos científicos (Cerrutti). Aunque en nuestro estereotipo un instrumento sea un bien mueble o una instalación, y las sustancias químicas parecen pertenecer presupuestariamente al apartado de fungibles, se puede argumentar que las sustancias químicas funcionan como instrumentos en el nivel molecular.

Dice Duhem al comienzo de La Théorie Physique (1906, p. 2) que «antes de aplicar un instrumento al estudio de un fenómeno, el experimentador, preocupado por la certeza, desmonta este instrumento, examina cada una de sus piezas..." Pero tampoco esto es siempre así. Igual que hay una variedad de tipos de instrumentos, hay una variedad de conductas apropiadas con respecto a ellos. $\mathrm{Si}$ todo el mundo, en la comunidad cientifica relevante, admite que un instrumen- 
to dado cumple su función anunciada, podemos decir que es neutral dentro de esta comunidad. Cuando este acuerdo existe, no es necesario que nadie abra el instrumento y se le puede considerar una "caja negra». Esta denominación, debida a Latour, no deja de ser caprichosa, cuando se supone que la cualidad principal de los instrumentos que se encuentran en este caso es precisamente la transparencia. En los casos de transparencia, las especificaciones funcionales del instrumento y la comprobación de que las cumple, naturalmente, son todo lo que se necesita para operar con él. Pero también, los instrumentos son «ideas in brassm, incorporan conocimiento. El constructor se basa en conocimientos diversos que pueden afectar a la operación, pero se podría añadir paradójicamente que a veces incorporan "conocimiento ignorado". No siempre es el caso que un instrumento que se usa y en cuyos resultados se confía esté explicado por una teoría satisfactoria. Exponer ese conocimiento es en general el motivo de abrir las cajas negras. La metodología de la "apertura de cajas negras" se extiende por distintos ámbitos de investigación.

El más evidente, el de la ingeniería inversa. Cuando ésta se practica por motivos de espionaje industrial, el ingeniero conoce las prestaciones o funciones que realiza el instrumento que desea desentrañar y trata de conjeturar cómo las realiza, a la vista de las interioridades del cacharro. Esto es, tiene que discernir los procedimientos causales relevantes para realizar las funciones especificadas del instrumento. Las películas de espías nos han familiarizado con la importancia de contar con los planos del ingenio y Nuestro Hombre en la Habana con los inconvenientes de tener que limitarse a ellos.

Los arqueólogos se encuentran a veces en el caso extremo de tener que decidir si un objeto hallado en una excavación es un instrumento o no (ver Burton y Simon (1996) y Peacock (1991)). Las "especificaciones" o descripciones funcionales plausibles para una lasca de pedernal no pueden diferenciar un origen artificial de uno natural. Se puede cortar o raspar con demasiadas cosas. El arqueólogo se ve obligado a descender del nivel de la descripción funcional por motivos, sin embargo, parecidos a los del espía industrial: está claro para qué podría servir y cómo podría hacer ese papel una lasca, pero tiene que averiguar mediante qué proceso se obtiene. Además, tiene que encontrar en el objeto rastros de la manipulación humana: estrías, particularidades del punto de fractura, etc.

Otras veces, los rasgos físicos del objeto hacen evidente su origen cultural, pero los papeles funcionales posibles no son restringidos lo suficiente por la con- 
figuración física. $\mathrm{El}$ arqueólogo puede restringir las especificaciones funcionales que se le ocurren o "descubrirlas» atendiendo a propiedades que ayuden a insertar el objeto en la cultura a la que pertenece. Para hacerlo tiene que imaginar un entorno en el que actúa ese instrumento. Ese entorno es un mundo posible que a veces no es el mundo real. Una máquina del movimiento perpetuo solo merece esa descripción en un mundo en el que no valgan las leyes de la termodinámica.

En la ciencia ficción son extremadamente corrientes los aparatos alienígenas de los que se sabe qué hacen, pero no cómo lo hacen. Lo único que se sabe de cómo funciona el teletransportador de materia de Star Trek es que funciona perfectamente. También son corrientes los aparatos de los que no se sabe ni una cosa ni la otra, pero no vamos a discutir las secuencias iniciales de Stargate.

Otra posibilidad respecto a los instrumentos es la sobreexplicación: Rasgos de difícil explicación o casuales de un artefacto pueden ser objeto de explicaciones excesivas que atribuyan demasiadas intenciones o demasiados conocimientos a quienes los fabricaron.

La psicología y la neurología, por la naturaleza de sus intereses, no han tenido más remedio que servirse de la metodología de la apertura de las cajas negras. Los psicólogos conductistas decidieron considerar el cerebro como una caja negra no porque estuviera claro cómo funciona, sino por todo lo contrario. En las objeciones que estos psicólogos oponían a la psicología mentalista tradicional podemos reconocer un precedente del "círculo hermenéutico" y del «regreso del experimentador». Los psicólogos conductistas advertían que cuando atribuimos un estado interno mental a un organismo no tenemos constancia neurológica de ese estado, porque los términos mentales no refieren a estados neuronales. De manera que nuestro indicio para la atribución es la conducta del organismo. Ahora bien, los estados mentales son supuestamente la explicación de la misma conducta que sirve como indicio de su presencia.

Cuando no se sabe cómo funciona ni qué hace exactamente una caja negra, una actitud de prudencia lleva a contar qué hace bajo qué circunstancias, y esto en un lenguaje tan neutral teóricamente y tan pormenorizado como sea posible. Estas descripciones, cuando se trataba de la conducta de los organismos, las llamaban los psicólogos "moleculares" en oposición a las descripciones molares, formuladas en términos más generales y que conllevan, por lo tanto, una doctrina de, al menos, las funciones que realiza el organismo. Bogen y Woodward afir- 
man con razón que los datos de la percepción de los experimentadores rara vez tienen un papel en la ciencia. Esto es tanto como decir que en la ciencia rara vez es necesario descender a descripciones muy moleculares, porque hay acuerdo sobre las descripciones en términos relativamente más molares.

En todos estos casos tenemos un instrumento o un organismo que actúa en un contexto o entorno material. Cuando sabemos poco acerca de ese objeto y sabemos poco acerca de sus relaciones con el entorno nos tenemos que conformar con describir qué estímulos o qué inputs del entorno sobre qué parte del instrumento tienen qué efecto sobre éste, o qué efectos sobre el entorno tienen las operaciones del objeto.

Cuando los psicólogos volvieron a atreverse a hablar acerca de la estructura de la caja negra buscaron varios medios para asegurarse de que las descripciones de los dispositivos en términos de sus funciones no incurrieran en el círculo hermenéutico. Los principales recursos que emplearon fueron los siguientes: Por una parte emplearon la metáfora del ordenador. En la psicología cognitiva prosperó por diversas razones la idea de que las acciones de la mente se pueden comparar a las de un ordenador. Se propuso que las teorías psicológicas tuvieran la forma de los diagramas de flujo que diseñan los programadores antes de proceder a la redacción de un programa. Se discutió en la filosofía de la mente si las mentes humanas son máquinas de Turing. Una de las ventajas de la metáfora del ordenador era garantizar que la teoría psicológica ofrecida bajo esa presentación era, al menos, instanciable, no era vacua.

Explicar una conducta como la salida de un ordenador evita el riesgo de la circularidad, pero no el de la infradeterminación empírica. Muchas máquinas de Turing pueden dar cuenta de la misma conducta.

En segundo lugar estudiaron los patrones del error en la realización de tareas para constreñir las posibles teorías computacionales de la conducta. Una máquina reconocedora de formas que tenga un patrón de equivocaciones inhumano no instancia una buena teoría del reconocimiento humano de formas.

En tercer lugar, aunque el objetivo está lejos de ser alcanzado, intentan compatibilizar y relacionar las teorías sobre diferentes tareas para construir una teoría unificada de la mente. Eso da a los mecanismos hipotetizados para cada tarea un carácter menos ad hoc. 
Un último procedimiento que se emplea en diferentes ocasiones, es la fragmentación de la caja negra. Los neurofisiólogos usaron en tiempos el método de la ablación del cerebro como método realmente desesperado: se corta un trozo, se ve qué deja de hacer el organismo y se sabe que ese fragmento desempeña algún papel en la realización de la función. Los psicólogos idean teorías subpersonales en las que las funciones, por ejemplo cognitivas, son realizadas por homúnculos. En química orgánica, cuando se desea profundizar en la constitución molecular desconocida de una enzima, se interrumpe la operación de ella sobre su sustrato y se ve qué productos parciales tiene la reacción. Estos procedimientos tienen la virtud de romper el círculo o el regreso del experimentador o de rellenar la relativa vacuidad de la descripción funcional. Ponen de manifiesto cómo se comprueba que una descripción funcional de un órgano o de una reacción química no es vacua porque se puede construir a partir de una descripción funcional de más bajo nivel de una parte del órgano o de la reacción operando en un entorno.

Cuando tenemos una teoría clara de las propiedades del entorno relevantes para la función que nos interesa de un organismo, entonces desaparecen las perplejidades metodológicas y el regreso del experimentador. Dawkins ha sacado mucho partido de las semejanzas entre organismos y artefactos. Las páginas que dedica en The Blind Watchmaker a los sistemas de ecolocalización de los murciélagos hacen evidente que el conocimiento de las propiedades del sonido permite sugerir y contrastar hipótesis sobre la constitución funcional de la detección y producción de sonidos de los murciélagos y sobre el papel de los órganos involucrados en estas tareas. También ha notado que existe una variedad de niveles de generalidad en la descripción de los componentes que en un artefacto o en un organismo realizan una función.

Dennett (1990), Hoffstadter y otros filósofos de la mente se han ocupado de cómo se constituyen entidades correspondientes a una ontología más general a partir de otras pertenecientes a otras más concretas. Aristóteles en De Anima ${ }^{3}$ ya advertía que el rubor del rostro puede ser explicado desde el punto de vista del físico y desde el punto de vista del dialéctico. El primero explica que se ha producido un aflujo de sangre y el segundo que la causa del enrojecimiento fue la vergüenza. Esta distinción de niveles de descripción y de explicación ha sido

\footnotetext{
${ }^{3} 403 \mathrm{a}-\mathrm{b}$
} 
reformulada varias veces en la filosofía de la mente o de la psicología. Fodor también encuentra niveles de explicación semejantes a los de Aristóteles, porque la explicación del nivel característicamente psicológico es, según Fodor (1968), una explicación funcional, emparentada con la explicación teleológica del dialéctico de Aristóteles.

Lo que Dennett intenta hacer es relacionar los diferentes niveles de descripción y de explicación. Hoffstadter y Dennett encuentran que un objeto u organismo o dispositivo que puede ser descrito en términos físicos puede tener una actuación regular dentro de un entorno, y que esa actuación regular permite designar esa entidad con un término de nivel superior, muchas veces funcional, porque indica lo que ese objeto hace en el entorno dado. Cuando lo que tenemos es una descripción funcional del objeto podemos desear conocer la constitución física y los mecanismos causales que producen la actuación funcionalmente descrita del objeto. Sin embargo, si tenemos un objeto que parece desempeñar una función, pero no sabemos cuál exactamente, la hipótesis inicial acerca de cómo la desempeña es, desde luego, que la desempeña bien. Incidentalmente, esta exigencia metodológica ha dado pie a muchas discusiones absurdas sobre el eufuncionalismo, degeneración cargada de valores, del funcionalismo en, por ejemplo, la sociología y la antropología. En la química orgánica es frecuente designar una enzima por lo que hace en un contexto dado: se designa indicando la sustancia sobre la que opera, lo que le hace y se acaba en "asa", y luego se busca su constitución molecular.

En "Géneros Naturales» resume Quine la estrecha conexión entre leyes científicas y géneros naturales: «la noción de género... es crucialmente relevante con respecto a la noción de disposición, al condicional subjuntivo y a los enunciados causales singularesm. ${ }^{4} \mathrm{Si}$ la generalización que podamos establecer acerca de la actuación de un organismo o de un instrumento en un tipo de situaciones soporta condicionales subjuntivos, entonces ese organismo $o$ artefacto constituye un género natural según el criterio de Quine. Las leyes relativas a un género son generalizaciones sobre lo que las cosas pertenecientes a él hacen en ciertas situaciones. Los motivos que se aducen para conceder a una generalización la dignidad de ley son variados y tienen diferente fuerza. Entre estos motivos está que la regularidad sea constante y, mejor aún, que pueda ser explicada por una teoría satisfactoria.

\footnotetext{
${ }^{4}$ p. 169 de la versión en español.
} 
Un género natural puede, además, ser establecido si tiene comportamientos regulares en contextos de tipos diferentes, y más aún si ayuda a fundar una conexión teórica entre esos tipos diferentes de situaciones.

Un instrumento es reconocido, de manera semejante a como lo es un género natural, si su comportamiento en un tipo de situaciones posee una regularidad constante. Mejor aún si hay una teoría satisfactoria de ese comportamiento. Los datos que suministra un instrumento son considerados fiables si el mecanismo causal por el que el instrumento ofrece los datos tiene una regularidad reconocible, y también si esos datos se pueden obtener mediante procedimientos que involucran secuencias causales de tipos diferentes.

Estos criterios de fiabilidad de los datos pueden entrar en conflicto porque no dependen en exclusiva de consideraciones lógicas, sino de cuestiones de hecho y del conocimiento sobre ellas. Acerca de ellos han polemizado Culp y Hudson. La primera ha invocado el concepto de robustez de los datos, de manera que un conjunto de datos es preferible en proporción a su robustez, caracterizándose esos datos por la circunstancia de haber sido obtenidos mediante técnicas experimentales diferentes que involucran procesos causales diferentes. Culp ha argumentado su posición basándose en un análisis del descubrimiento de que los mesosomas, por un tiempo considerados pequeńos órganos celulares, son en realidad creaciones de las técnicas experimentales que los identificaban. En un análisis a contrapelo del mismo caso histórico, Hudson cree haber hallado que la noción de robustez tal como la entiende Culp no desempeñó ningún papel, sino que fue decisiva la confianza en un proceso experimental fiable, esto es estable y bien comprendido. De acuerdo con Hudson un proceso que tenga estas cualidades puede proporcionar datos preferibles a los que ofrecen varios procesos independientes pero menos fiables.

Los instrumentos filosóficos característicos de lo que Kuhn ha llamado "ciencia baconiana" son construidos en servicio del estudio de unos fenómenos mal conocidos. Boyle, por ejemplo, renuncia al ideal demostrativo de certeza de las ciencias matemáticas y elogia la moderada finalidad de obtener un conocimiento probable. ¿Cuál es el terreno firme en el que se pueden discutir unos fenómenos acerca los cuales no hay una teoría general compartida? Naturalmente, las descripciones más minuciosas de los hechos involucrados en un lenguaje compartible. Y un instrumento que producía fenómenos inesperados o controvertidos debía ser dibujado y descrito con grandes pormenores. Si no se conoce, 
o es debatible, cuál es la actuación del instrumento sobre un entorno dado, pero su inventor insiste en que es capaz de producir cierto efecto, entonces está obligado a ofrecer una descripción extremadamente detallada del instrumento, relativamente molecular. Cuando un instrumento "soporta" condicionales contrafácticos sobre su comportamiento en circunstancias dadas, y todo el mundo admite esos condicionales, no hay por qué recurrir a descripciones detalladas: basta con anunciar que el instrumento es un termómetro, o una bomba de vacío, o lo que sea, y es legítimo representarlo mediante un diagrama idealizado, como propone Duhem.

Además de descripciones y dibujos minuciosos de los instrumentos y de las situaciones experimentales, en la ciencia baconiana se produce otro fenómeno característico, como es la convocatoria de testigos fiables. Parece evidente que el método de convocar testigos sirve el propósito de dar fe de que acontecimientos que podrían no haber ocurrido, en realidad ocurrieron. Cuando no se sabe lo que puede pasar es conveniente que haya testigos de lo que pasó si es que hay que tomar decisiones sobre esa base.

En un estudio sobre la bomba de vacío de Boyle, Shapin conecta el papel del testimonio para constituir un hecho en ciencia con la ideología clasista de la época. Destaca que en materia de experimentación, según Boyle, los testigos convocados para certificar un acontecimiento deberían ser personas de fiar, es decir, personas veraces y conocedoras, al menos en algún grado, de asuntos de filosofía natural. Observa Shapin que las condiciones del testimonio que exige Boyle coinciden con las condiciones que se exigían en el derecho "procesal" inglés, y llama "retóricas" a las presentaciones y dibujos extremadamente fieles que ofrece de la configuración y operación de la bomba. Leyendo a Shapin no puede uno menos que recordar al antropólogo del chiste, que ante el cráter del Kilawea ofrecía una pieza de mitología local: «El Kilawea, del que dice la leyenda que los que cayeron al cráter nunca, nunca regresaron». Si rasgos culturales tan poco pintorescos, que se explican con sencillez en términos intencionales, son objeto de otra explicación, también intencional, ¿qué motivos tenemos para pensar que la segunda es más iluminadora?

Un caso semejante, en cuanto al tipo de representación, al de la bomba de aire es el del calorímetro de Lavoisier. En una ciencia en construcción, con doctrinas alternativas ninguna de las cuales está en posesión de todos los ases aparece un aparato que, según una de las teorías enfrentadas, hace algo inefable, es 
decir, algo que no puede ser concebido en sus términos. El primer movimiento de Lavoisier es decir que ha construido una máquina: el vocabulario compartido entre teorías rivales está en niveles muy bajos o muy altos y, por lo tanto Lavoisier ofrece un dibujo muy detallado y un nombre muy general. Conforme su teoría química se consolida, Lavoisier se atreve a darle un nombre a su máquina, da una descripción funcional de lo que hace. Dicho sea de paso, la teoría química de Lavoisier consiguió éxitos independientes del éxito del calorímetro que, en vista de objeciones técnicas poderosas, fue abandonado como instrumento de la investigación científica.

Los recursos que se emplean para abrir una caja negra, o para constituir un género natural no están garantizados. No hay método científico que asegure la obtención de un resultado de buena teoría. Pero el círculo vicioso que envuelve géneros y leyes o instrumentos y datos se puede romper mediante varios procedimientos, algunos han sido mencionados.

"Detector", "termómetro", "refuerzo», «interruptor", "barbitúrico" etc. son términos funcionales. Pueden aparecer como nombres de entidades cuando éstas son descritas en un nivel de generalidad apropiado. Un nivel de generalidad es apropiado cuando en él se pueden formular generalizaciones que soporten contrafácticos. Para que una sustancia cuente como barbitúrico basta con que sea cierto que si alguien la toma le dé sueño. Para que un término quede legitimado como designador de un género natural basta con esto, pero la mayoría de las veces se desea una explicación de por qué el refuerzo refuerza o el barbitúrico duerme o el detector detecta. Esto es semejante a pedir una explicación de un enunciado universal relativo al comportamiento de la entidad del caso. Cuando procedemos a dar esa explicación el género natural puede romperse: La virtus dormitiva del opio puede obedecer a mecanismos fisiológicos diferentes de los de un ensayo de filosofía.

Cuando se tiene un género reconocido se puede hacer ciencia normal buscando nuevas leyes acerca de ese género. El regreso del experimentador no es sino la versión, en el mundo de los instrumentos, del familiar problema de establecer géneros naturales y leyes simultáneamente. 


\section{BIBLIOGRAFIA}

ARISTÓteles, De Anima.

J. BOGEN y J. WOODWARD, «Observations, Theories and the Evolution of the Human Spirit", Philosophy of Science, n. ${ }^{\circ}$ 59, 1992.

J. Burton y A. W. Simon, «A Pot is Not a Rock: A Reply to Neff, Glasnock, Bishop and Blackman, American Antiquity, vol. 61, n. ${ }^{\circ}$ 2, 1996.

L. CERRUTTI, "Chemicals as Instruments. A Language Game», International Journal for the Philosophy of Chemistry, vol. 4, n. ${ }^{\circ} 1,1998$.

H. Collins, Changing Order, Londres, 1985.

S. CulP, "Objectivity in Experimental Inquiry: Breaking Data-Technique Circles", Philosophy of Science, vol. 62, no 3, 1995

D. DennetT, "The Interpetation of Texts, People and Other Artifacts", Philosophy and Phenomenological Research, vol. 1, suplement, 1990.

P. Duhem, La Théorie Physique, Son Object, Sa Structure, 1906 http://www.abu.org. Edición de P. Cubaud.

J. FODOR, Psychological Explanation, Nueva York, 1968.

R. Hudson, "Mesosomes: A Study in the Nature of Experimental Reasoning", Philosophy of Science, vol. 66, n. ${ }^{\circ} 2,1999$.

T. S. KuHN, «La Tradición Matemática y la Tradición Experimental en el Desarrollo de la Física", en T. S. Kuhn, La Tensión Esencial (trad. de R. Helier), F.C.E., 1ª reimpr. en España, Madrid, 1983.

E. PEACOCK, «Distinguishing between Artifacts and Geofacts: A Test Case from Eastern England", Journal of Field Archaeology, vol. 18, n. ${ }^{\circ} 3,1991$.

W. v. QUINE, "Géneros Naturales», en La Relatividad Ontológica y Otros Ensayos (trad. de M. Garrido y J. Ll. Blasco) Tecnos, Madrid, 1974.

L. ROBERTS, «A Word and the World. The Significance of Naming the Calorimeter", Isis, 82, 1991. 
S. SHAPIN, "Pump and Circumstance: Robert Boyle's Literary Technology», Social Studies in Science, vol. 14, n. ${ }^{\circ} 4,1984$.

M. SPECTOR, "Theory and Observation (I)", The British Journal for the Philosophy of Science, vol. 17, 1966.

G. L' E. TURNER, «Interpreting the History of Scientific Instruments", en R. G. W. Anderson, J. A. Bennett y W. F. Ryan (eds.), Making Instruments Count, Aldershor, 1993. 